\title{
Computational Method for Forensic Verification of offline Signatures
}

\author{
Vaibhav Saran ${ }^{1}$, Suneet Kumar ${ }^{2}$,Syeed Ahmed ${ }^{3}$ \& A.K.Gupta ${ }^{4}$ \\ 1,2 and 4(Department of Forensic Science, SHIATS-DU, India) \\ 3 (Central Forensic Institute, Bhopal, India)
}

\begin{abstract}
Signature verification models based on personal model have been reported by many researchers in past but the method proposed here is a forensic document examination approach using computational methods, unlike other models which require a considerable number of genuine signatures of the same writer to correctly train the model. This paper proposes a forensic signature verification approach making a robust verification system even when few samples per writer are available. The efficiency of the proposed method is based on results of 150 writers with 10 signatures of each writer.
\end{abstract}

Keywords: Neural Network Classifier, offline signature verification system, SVM,

\section{Introduction}

Pattern recognition for offline signature or handwritten text verification has gained attention of most of the researcher in last few decades. Most of the research proposed for offline signature verification system is based on the personal model which requires a large data base of signatures to train the model. Previous work have reported that major drawback of such model was the need of trained model as every time a new signature or sample from new writer has to be included in bulk to the model to make a reliable model [1].

Forensically a signature is verified by visually comparing the suspected with the reference by a skilled forensic handwriting expert. However the authenticity of the questioned handwriting or signature is estimated by evaluating the characteristic features of handwriting or signatures and their similarities and differences within the suspected and the reference samples.

Computational methods can assist the forensic handwriting experts in evaluating the probability of the evidence under investigation that whether the questioned signature is an authentic signature normally used by the reference writer; or the questioned signature is the product of a forgery process. The examiner evaluates the features and interprets the results based on the similarities or dissimilarities observed within the suspected and the reference samples of signatures. Since handwriting including signatures are a product of a behavioral process and can be manipulated and hence in signature verification research, a perfect match does not treated as genuine as a perfect match can occur only if it is traced. And the differences observed in them cannot be treated as forged because slight changes can occur due to natural variation.

In this paper a computational method based on forensic document approach for signature verification is proposed which computationally calculates the features that is observed by a handwriting expert and the signature is verified computationally.

\section{Methodology}

A data set of 150 signatures obtained from different writers including 100 control signatures with known authorship and 50 suspected signatures which were forged by different writers were scanned through high resolution scanner at $600 \mathrm{dpi}$ and were used for further analysis.

\subsection{Image Preprocessing}

Digital images are prone to a variety of types of noise which may be the result of errors in the image acquisition process that result in pixel values that do not reflect the true intensities of the real scene. The scanned images were preprocessed to remove any noise present in it using Linear Filtering using Gaussian filters.

\subsection{Image Segmentation}

The preprocessed image is segmented using Otsu method [2] which chooses the threshold to minimize the intra class variance of the black and white pixels. Multidimensional arrays are converted to 2-D arrays using reshape. 


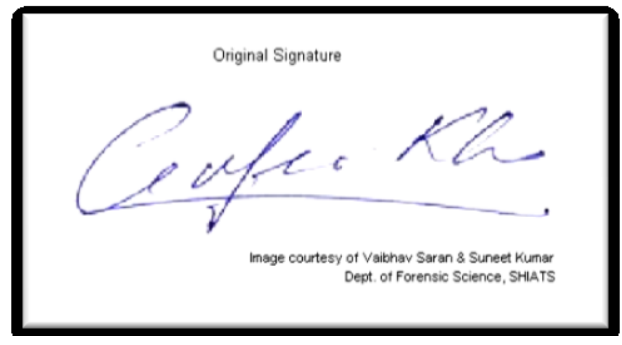

Figure 1: original signature image

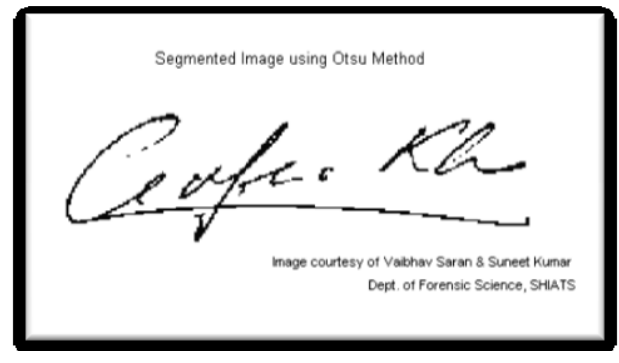

Figure 2: segmented image

\subsection{Extraction of Signature Verification Features}

The segmented image is then subjected for feature extraction, which will be computed in order to verify the signatures the major significant features like Binarization, Horizontal Position, Vertical Position, Height and Width, Slant, Alignment, Spacing and Angle between strokes were extracted from the binarized image obtained after segmentation[1,3].

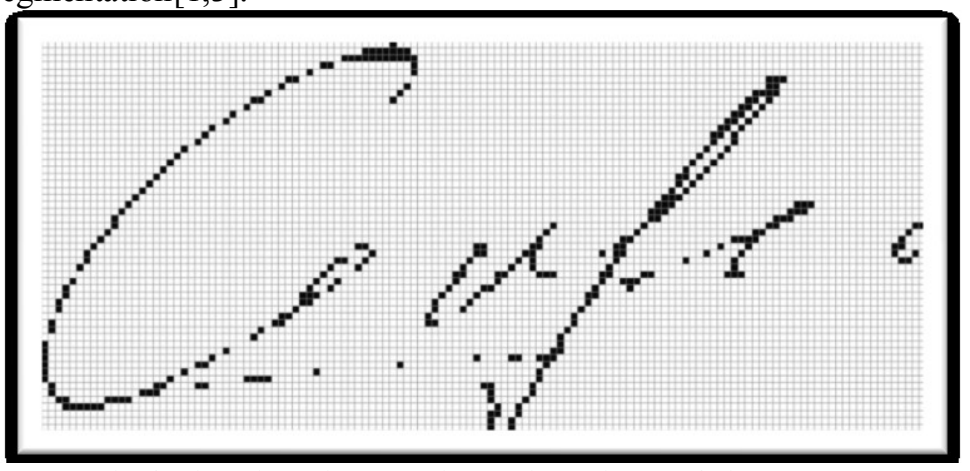

Fig 3 binarized image displaying pixels of signatures

\subsection{Experimental Setup for Classification}

All the segmented images were assembled to make a dataset of 150 signatures. The data was converted to a uniform format and saved in a single folder. For every signature number of strokes and their $\mathrm{x}, \mathrm{y}$ coordinates were extracted using impixelinfo command of matlab, the coordinates for every sample were carefully checked for any errors and labeled. The coordinates thus obtained were computed to calculate the values for the parameters selected for this study using their respective formula as shown in Table 1.

TABLE 1: Features selected for present study

\begin{tabular}{|c|c|c|c|}
\hline Sr. No & Characteristic Observed & Formula Used for calculation & Classified as \\
\hline 1 & Slant & $\Theta=\left(y_{1}-y_{2}\right) /\left(x_{1}-x_{2}\right)$ & $\begin{array}{l}\text { Right Slant if } \Theta>90 \\
\text { Left Slant if } \Theta<90 \\
\text { Vertical Slant if } \Theta=90\end{array}$ \\
\hline 2 & Size of letters & $\begin{array}{l}\qquad \text { Size }=\sqrt{\left(x_{1}-x_{2}\right)^{2}+\left(y_{1}-y_{2}\right)^{2}} \\
\text { Average Vertical distance of letter on } \mathrm{x}, \mathrm{y} \text { plane } \\
\text { calculated by distance formula }\end{array}$ & $\begin{array}{l}\text { Small } \\
\text { Medium } \\
\text { Large } \\
\text { (Based on number of Pixel) }\end{array}$ \\
\hline 3 & Alignment & $\begin{array}{l}\text { Tan } \Theta=\text { Base/Hypotenuse } \\
\text { The angle between a line and the } \mathrm{x} \text {-axis is measured } \\
\text { counterclockwise from the part of the } \mathrm{x} \text {-axis to the } \\
\text { right of the line. }\end{array}$ & $\begin{array}{l}\text { Horizontal if } \Theta=180^{\circ} \\
\text { Uphill if } \Theta<180^{\circ} \\
\text { Down Hill if } \Theta>180^{\circ}\end{array}$ \\
\hline 4 & Spacing & $\begin{array}{l}\text { Average Horizontal Distance between letters, words } \\
\text { and lines on } \mathrm{x}, \mathrm{y} \text { plane calculated by } \\
\text { Space }=\sqrt{\left(x_{1}-x_{2}\right)^{2}+\left(y_{1}-y_{2}\right)^{2}}\end{array}$ & $\begin{array}{l}\text { Moderate } \\
\text { Narrow } \\
\text { Wide } \\
\text { (Based on number of Pixel) }\end{array}$ \\
\hline 5 & Angle of Strokes & $\Theta=\tan ^{-1}\left(m_{2}-m_{1}\right) / 1+\left(m_{2} * m_{1}\right)$ & $\begin{array}{l}\text { Acute if } \Theta<90 \\
\text { Obtuse if } \Theta>90 \\
\text { Right Angled if } \Theta=90\end{array}$ \\
\hline 6 & Aspect Ratio of Signature & Aspect ratio $=$ Width $(\mathrm{W}) /$ Height $(\mathrm{L})$ & $\begin{array}{l}\text { Tall }(\mathrm{L}>\mathrm{W}) \\
\text { Wide }(\mathrm{W}>\mathrm{L})\end{array}$ \\
\hline
\end{tabular}




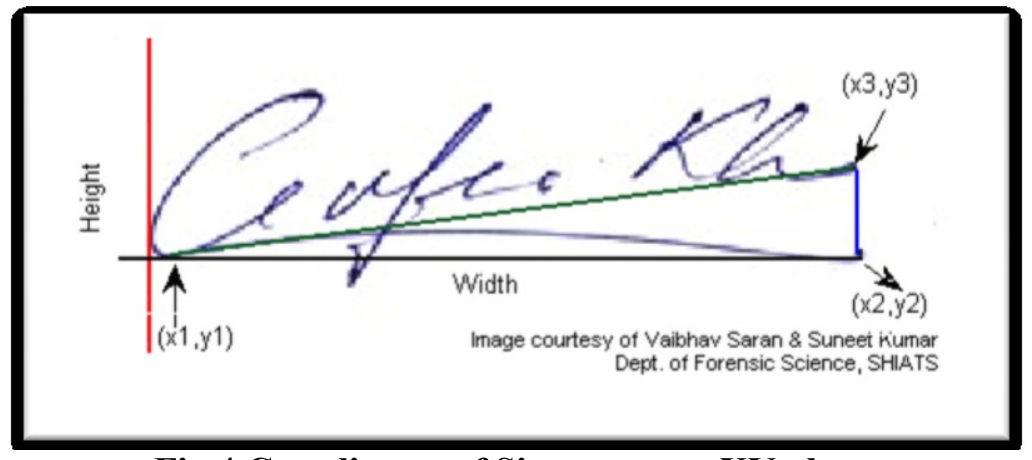

Fig 4 Coordinates of Signatures at XY plane

\section{Result:}

The model tested with 100 control signature samples for each parameter and validated by analyzing 50 suspected signatures by taking their mean values, the results obtained by the computational methods were highly significant and showed an average $96.7 \%$ and $94.5 \%$ of accuracy for control and suspected sample respectively as reported in Table 2.

TABLE 2: Accuracy percent of positive identification of features in signatures

\begin{tabular}{|l|l|l|l|l|l|}
\hline \multirow{2}{*}{ Sr. No } & \multirow{2}{*}{ Feature } & No. of Tested Signatures & \multicolumn{2}{|c|}{ Accuracy (\%) } \\
\cline { 3 - 6 } & & Control & Suspected & Control & Suspected \\
\hline 1 & Slant of Signature & 100 & 50 & 98 & 92 \\
\hline 2 & Size & 100 & 50 & 98 & 94 \\
\hline 3 & Alignment & 100 & 50 & 96 & 95 \\
\hline 4 & Spacing & 100 & 50 & 92 & 92 \\
\hline 5 & Angle of Strokes & 100 & 50 & 98 & 98 \\
\hline 6 & Aspect Ratio & 100 & 50 & 97 & 95 \\
\hline
\end{tabular}

\section{Conclusion:}

The computational method of measuring pixel values of signatures based on coordinate geometry concepts for forensic significance as shown in this paper allows distance and angle based classification of signatures with relatively better accuracy as compared to various model that have been proposed by several researchers. The method proposed here is however based on forensic document examination approach and will certainly aid as an important tool for verification of signatures in comparatively less time than the traditional methods used in forensic labs and with greater accuracy.

\section{References:}

[1] Cesar R. Santos, Flávio Bortolozzi, Luiz S. Oliveira, Edson Justino, 2007 Off-line Signature Verification Based on Forensic Questioned Document Examination Approach Pontifical Catholic University of Parana, SAC'07, March 11-15, Seoul, Korea.

[2] Srihari, A. S., Cha, H. Arora, S. Lee, 2001. "Individuality of Handwriting: A Validity Study", Proc. ICDAR"01, Seattle (USA), pp 106-109.

[3] Oliveira L. S., Justino, E., Freitas, C., and Sabourin, R. 2005, The Graphology Applied to Signature Verification, $12^{\text {th }}$ Conference of the International Graphonomics Society (IGS 2005), pages 286-290. 